\title{
Placental miRNA expression profiles are associated with measures of infant neurobehavioral outcomes
}

\author{
Matthew A. Maccani ${ }^{1,2}$, James F. Padbury ${ }^{3,4}$, Barry M. Lester ${ }^{3-5}$, Valerie S. Knopik ${ }^{1,6}$ and Carmen J. Marsit ${ }^{7,8}$
}

BACKGROUND: A growing body of research suggests that the intrauterine environment influences fetal neurodevelopment by altering the functional placental epigenome. A number of microRNAs (miRNAs) are expressed in the placenta, may be sensitive to dysregulation by environmental exposures, and are associated with adverse pregnancy outcomes. Our study aimed to identify the relationships between placental miRNA expression and newborn neurobehavior.

METHODS: We examined the association between the expression of miR-16, miR-21, miR-93, miR-135b, miR-146a, and miR-182 in total RNA from the placentas of 86 term infants as measured by quantitative real-time PCR and newborn neurobehavioral outcomes as assessed using the NICU Network Neurobehavioral Scales (NNNS).

RESULTS: Bivariate analysis revealed that placental miR-16 expression is negatively associated with attention score $(P=$ 0.006), whereas expressions of both miR-146a and miR-182 are positively associated with quality of movement score $(P=$ 0.016 and $P=0.016$, respectively). Controlling for potential confounders, high miR-16 expression is significantly associated with reduced attention score $(P=0.04)$, and high miR-146a and miR-182 expressions are significantly associated with increased quality of movement score ( $P=0.04$ and $P=0.01$, respectively). CONCLUSION: These results suggest that placental miRNA expression is associated with early neurobehavioral outcomes and miRNAs in the placenta may contribute to the developmental origins of infant neurobehavior.

$\mathbf{T}$ he period of fetal development is a critical period during which changes in the intrauterine environment can have significant consequences for future health. The early focus of the field of fetal programming was on associations between prenatal exposures and metabolic diseases and related disorders such as coronary heart disease, obesity, and diabetes (1). More recently, research has investigated the influence of the prenatal environment on neurobehavioral outcomes such as schizophrenia, depression, inhibitory control, and attentiondeficit/hyperactivity disorder (2).
As a highly active regulator of metabolic and endocrine status in utero, the placenta plays an important role in modulating the fetal environment and in providing appropriate hormones and peptides needed for fetal growth and development. Because of its role in modulating the influence of prenatal exposures and its major role in production of important neuropeptides such as corticotropin-releasing hormone and thyrotropin-releasing hormone, the placenta has been considered by some to be the "third brain" (3). In this role, the placenta links the developed maternal brain and developing fetal brain to the influence of environmental insults (4), some of which may have life-altering effects on the newborn.

The importance of epigenetic regulation has gained prominence in investigations of molecular mechanisms by which the prenatal environment can influence the placenta (5). One important mode of epigenetic regulation is expression of microRNAs (miRNAs), small noncoding RNAs that are involved in posttranscriptional gene regulation. Through posttranscriptional gene regulation and tissue-specific expression and function, miRNAs have been shown to regulate a number of key cell processes, including invasion, migration, proliferation, and death $(5,6)$, and to be critical regulators of embryonic development. miRNAs have been shown to be expressed in the placenta (7), and dysregulated placental miRNA expression has been associated with prenatal exposures $(8,9)$, pregnancy outcomes $(7,10,11)$, and fetal growth (12). miRNAs have also been shown to be associated with a variety of neurobehavioral outcomes, including pathways that may lead to neurobehavioral dysfunction (13) or addiction (14).

Given the growing body of literature suggesting that the functional epigenome of the placenta may be associated with infant neurobehavioral outcomes $(15,16)$ and that placental miRNA expression may be both dysregulated by the intrauterine environment (8) and associated with fetal outcome $(12,17)$, we hypothesized that placental miRNA expression would be associated with infant neurobehavioral outcomes as assessed by the NICU Network Neurobehavioral Scales (NNNS) (18).

\footnotetext{
'Division of Behavioral Genetics, Rhode Island Hospital, Providence, Rhode Island; ${ }^{2}$ Center for Alcohol and Addiction Studies, Brown University, Providence, Rhode Island; ${ }^{3}$ Department of Pediatrics, Women \& Infants Hospital of Rhode Island, Providence, Rhode Island; ${ }^{4}$ Alpert Medical School, Brown University, Providence, Rhode Island; ${ }^{5}$ Brown Center for the Study of Children at Risk, Women \& Infants Hospital of Rhode Island, Providence, Rhode Island; ${ }^{6}$ Department of Psychiatry and Human Behavior, Brown University, Providence, Rhode Island; ${ }^{7}$ Department of Pharmacology and Toxicology, Dartmouth Medical School, Hanover, New Hampshire; ${ }^{8}$ Epidemiology and Biostatistics Section, Department of Community and Family Medicine, Dartmouth Medical School, Hanover, New Hampshire. Correspondence: Carmen J. Marsit (carmen.j.marsit@dartmouth.edu) 


\section{Articles $\mid$ Maccani et al.}

\section{RESULTS}

A total of 86 human placentas were analyzed for the expression of six candidate miRNAs previously shown to be expressed in the placenta, associated with in utero exposures (8) and fetal outcomes (12), and involved in regulating cell processes: $m i R-16, m i R-21, m i R-93, m i R-135 b, m i R-146 a$, and $m i R-182$. Demographic information is described in Table 1. Clinical characteristics and NNNS summary scores are presented in Table 2. Based on the a priori design of the cohort, the study population is overrepresented by small for gestational age and large for gestational age infants. The mean birth weight percentile calculated using the method of Fenton (19) was 44.2\%. Table 2 also provides descriptive statistics of the summary scores for infant attention, arousal, excitability, hypertonicity, stress/abstinence, and quality of movement as measured by the NNNS. Because the size of the study population was modest as compared with the number of domains evaluated, we focused our examinations on these six NNNS summary scores. This is consistent with prior reports that have noted that these summary scores were the most reflective of underlying at-risk profiles and are the most sensitive to various influences of the perinatal environment (20).

Supplementary Table S1 online describes the expression of the six candidate miRNAs in all of the 86 samples, based on quantitative real-time PCR and absolute quantification from a standard curve. As observed previously (12), placental miRNA data were not normally distributed. Therefore, the miRNA expression profiles were split into quartiles $(\mathrm{Q} 1=$ lowest quartile, 0-25\%; Q2 = lower-middle quartile, 26-50\%; Q3 = uppermiddle quartile, 51-75\%; Q4 = highest quartile, 76-100\%). NNNS summary scores were plotted by quartile of miRNA expression, and Kruskal-Wallis tests were used to assess differences in all six NNNS summary scores across quartiles of miRNA expression. Supplementary Table S2 online presents

Table 1. Demographics of the study population $(n=86)$

\begin{tabular}{lc}
\hline Demographic & \\
\hline Gender & \\
$\quad$ Male $(n, \%)$ & $51(41 \%)$ \\
$\quad$ Female $(n, \%)$ & \\
Maternal insurance & $50(58 \%)$ \\
$\quad$ Private $(n, \%)$ & $36(42 \%)$ \\
$\quad$ Not private $(n, \%)$ & \\
Maternal smoking during pregnancy & $5(6 \%)$ \\
$\quad$ Yes ( $n, \%)$ & $80(93 \%)$ \\
$\quad$ No ( $n, \%)$ & $29.1(6.09)$ \\
Maternal age in years (mean, SD) & \\
Delivery method & $54(63 \%)$ \\
$\quad$ Vaginal ( $n, \%)$ & $31(36 \%)$ \\
$\quad$ Cesarean section ( $n, \%)$ & $44.2(35.99)$ \\
Birth weight percentile ((mean, SD), \%) & $20(10.0)$ \\
Percentage maternal weight gained during pregnancy \\
$(($ mean, SD), \%)
\end{tabular}

the complete results of Kruskal-Wallis tests for differences of all six NNNS summary scores of interest across all six miRNAs measured. Analyses revealed that attention score significantly differed across quartiles of placental miR-16 expression $(P=$ 0.046 ), quality of movement score significantly differed across quartiles of miR-146a expression $(P=0.022)$, and quality of movement score significantly differed across quartiles of $m i R$ 182 expression $(P=0.027)$. Figure $1 \mathrm{a}-\mathrm{c}$ shows the distribution of infant NNNS score ( $y$-axis) by primary term human placenta miRNA expression quartiles ( $x$-axis) for these significant results.

Because the highest quartiles of miR-16 expression were associated with differential attention score, and the highest quartiles of $m i R-146 a$ and $m i R-182$ expression were associated with differential quality of movement score, we focused on the association between high expression (> median vs. $\leq$ median) of the miRNA and respective NNNS summary score. Tests revealed that high miR-16 expression was negatively associated with attention score $(P=0.006)$, high $m i R-146 a$ expression was positively associated with quality of movement score $(P=$ $0.016)$, and high $m i R-182$ expression was positively associated with quality of movement score $(P=0.016)$. These relationships are shown graphically in Figure 2a-c. Multivariable linear regression models were then used to examine the associations between high miRNA expression and NNNS summary scores, controlling for potential confounders. The results of these models are summarized in Table 3 and include effect sizes ( $\beta$ values) and statistical significance. After controlling for confounders, the linear regression analysis showed that high miR-16 expression was negatively associated with attention score $(\beta=-0.58, P=0.04)$, high miR-146a expression was positively associated with quality of movement score $(\beta=0.32$, $P=0.04)$, and high $m i R-182$ expression was positively associated with quality of movement score $(\beta=0.39, P=0.01)$.

\section{DISCUSSION}

miRNA expression has been associated with a variety of disease-associated or developmentally associated neurobehavioral outcomes $(13,14)$, and epigenetic mechanisms in the placenta

Table 2. Clinical characteristics and NNNS summary scores

\begin{tabular}{|c|c|c|c|c|c|c|}
\hline Variable & $n$ & Mean & SD & Median & Minimum & Maximum \\
\hline $\begin{array}{l}\text { Birth weight } \\
\text { percentile }\end{array}$ & 86 & 44.2 & 35.99 & 42.5 & 0 & 99 \\
\hline $\begin{array}{l}\text { Maternal } \\
\text { age (y) }\end{array}$ & 86 & 29.1 & 6.09 & 29.5 & 18 & 40 \\
\hline Attention & 86 & 3.8 & 1.13 & 3.64 & 1.57 & 6.17 \\
\hline $\begin{array}{l}\text { Quality of } \\
\text { movement }\end{array}$ & 86 & 4.05 & 0.68 & 4.17 & 2.17 & 5.33 \\
\hline $\begin{array}{l}\text { Stress } \\
\text { abstinence }\end{array}$ & 86 & 0.22 & 0.07 & 0.22 & 0.08 & 0.41 \\
\hline Arousal & 86 & 4.11 & 0.8 & 4.07 & 2 & 6 \\
\hline Hypertonicity & 86 & 0.29 & 0.68 & 0 & 0 & 3 \\
\hline Excitability & 86 & 4.58 & 2.99 & 4.5 & 0 & 13 \\
\hline
\end{tabular}



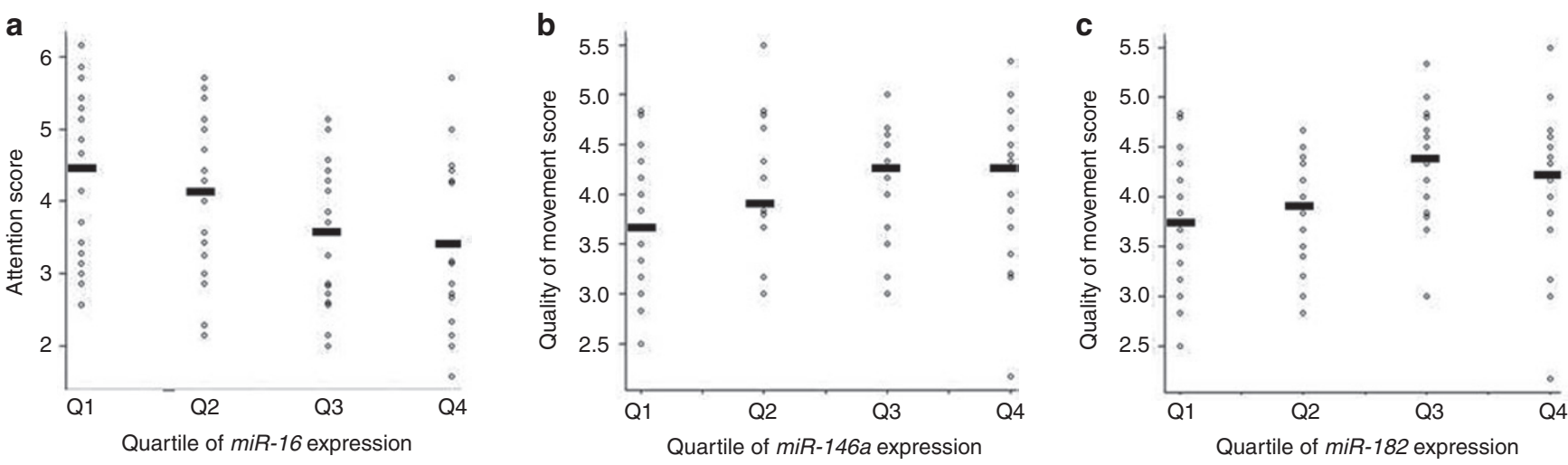

Figure 1. Distribution of infant NNNS score ( $y$-axis) by primary term human placenta miRNA expression quartile ( $x$-axis). Black bars indicate mean NNNS score within each quartile. (a) Placental miR-16 expression is negatively associated with attention score $(P=0.046)$, (b) placental miR-146a expression is positively associated with quality of movement score $(P=0.022)$, and (c) placental miR-182 expression is positively associated with quality of movement score $(P=0.027)$. miRNA, microRNA; NICU, neonatal intensive care unit; NNNS, NICU Network Neurobehavioral Scales.

a

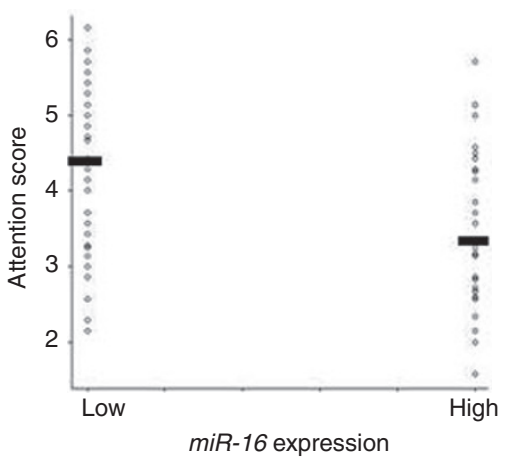

b

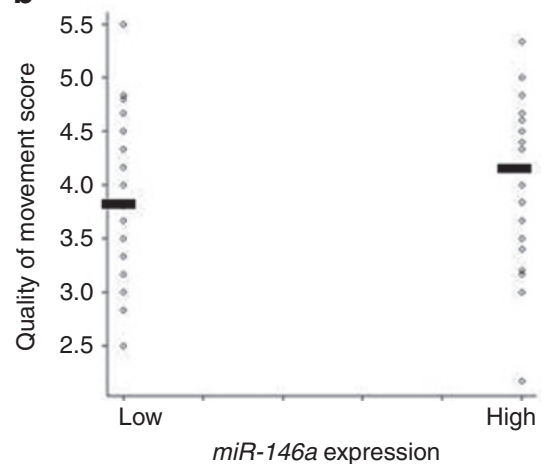

C

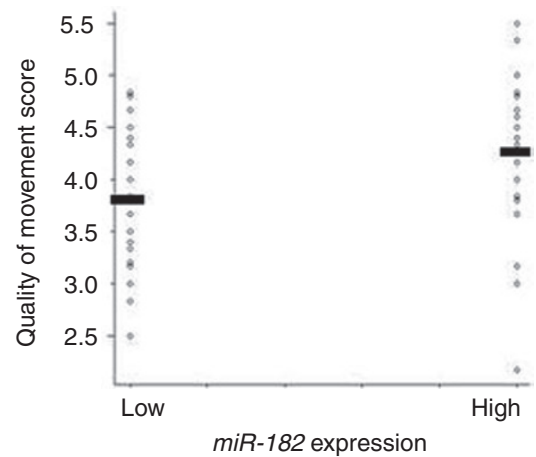

Figure 2. Distribution of infant NNNS score ( $y$-axis) by high-low miRNA expression groups ( $x$-axis). Black bars indicate mean NNNS score within each group. (a) Placental miR-16 expression is negatively associated with attention score $(P=0.006)$, (b) placental miR-146a expression is positively associated with quality of movement score $(P=0.016)$, and $(\mathbf{c})$ placental miR-182 expression is positively associated with quality of movement score $(P=0.016)$. miRNA, microRNA; NICU, neonatal intensive care unit; NNNS, NICU Network Neurobehavioral Scales.

have been associated with infant neurobehavioral outcomes $(15,16)$. However, to our knowledge, our analysis is one of the first to report associations between placental miRNA expression and infant neurobehavioral measures as assessed by the NNNS. Specifically, we have identified the associations of increased miR-16 expression with decreased infant attention score, increased miR-146a expression with increased infant quality of movement score, and increased miR-182 expression with increased quality of movement score.

The attention summary score scale describes an infant's response to auditory and visual stimulation and ability to maintain a quiet awake state (21). Infants with high attention summary scores exhibit good turning and following during orientation tests, showing a level of sustained alertness throughout. Low attention summary scores are reflective of minimal turning and following and briefer periods of alertness during the presentation of orientation items, indicative of an overall poor quality of response. Furthermore, a low attention score has been shown to reflect a low threshold for stimulation and has been frequently seen in infants who have been described as physiologically unstable (21). Our linear regression models, after controlling for potential confounders, suggested that high placental miR-16 expression was associated with a significant decrease in attention score of 0.58 units, reflective of an overall poor quality of response and reduced infant alertness.

The quality of movement summary score is an overall measure of motor control, including descriptions of an infant's smoothness, maturity, modulation of movement of the arms and legs, and startles and tremors (21). High scores suggesting good quality of movement are descriptive of infants who exhibit smooth movement with a low degree of jitteriness, tremors, and startles and relatively average amounts of spontaneous and elicited motor activity (21). Low scores suggesting an overall poor quality of movement describe infants who are predominantly jittery with a low degree of smoother movement of their arms and legs and with a great degree of tremors and startles, and a high overall level of activity (21). Our linear regression models controlling for potential confounders suggested that high placental miR-146a expression was associated with a significant increase in quality of movement score of 0.32 units, and high placental miR-182 expression was associated with a significant increase in quality of movement score of 0.39 units. Data suggesting that high miR-146a expression and $m i R-182$ expression are positively associated with higher 


\section{Articles | Maccani et al.}

Table 3. Linear regression models for the association between miRNA expression and NNNS score

\begin{tabular}{|c|c|c|c|c|c|c|}
\hline & \multicolumn{2}{|c|}{ Attention score } & \multicolumn{2}{|c|}{ Quality of movement score } & \multicolumn{2}{|c|}{ Quality of movement score } \\
\hline & Estimate & $P$ value & Estimate & $P$ value & Estimate & $P$ value \\
\hline \multicolumn{7}{|l|}{ miR-16 expression } \\
\hline High & -0.58 & 0.04 & - & - & - & - \\
\hline \multicolumn{7}{|l|}{ miR-146a expression } \\
\hline High & - & - & 0.32 & 0.04 & - & - \\
\hline \multicolumn{7}{|l|}{ miR-182 expression } \\
\hline Low & - & - & - & - & Reference & \\
\hline High & - & - & - & - & 0.39 & 0.01 \\
\hline $\begin{array}{l}\text { Maternal age in } \\
\text { years }\end{array}$ & -0.01 & 0.57 & 0.01 & 0.62 & 0.01 & 0.55 \\
\hline \multicolumn{7}{|l|}{ Maternal insurance } \\
\hline Public & Reference & & Reference & & Reference & \\
\hline Private & 0.52 & 0.07 & 0.14 & 0.40 & 0.20 & 0.24 \\
\hline $\begin{array}{l}\text { Relative pregnancy } \\
\text { weight gain }\end{array}$ & -0.75 & 0.56 & 0.38 & 0.62 & 0.46 & 0.55 \\
\hline \multicolumn{7}{|c|}{ Maternal smoking during pregnancy } \\
\hline No & Reference & & Reference & & Reference & \\
\hline
\end{tabular}

miRNA, microRNA; NICU, neonatal intensive care unit; NNNS, NICU Network Neurobehavioral Scales.

quality of movement scores may suggest that these two miRNAs may play a role in the pathway regulating fetal nerve and muscle development, with higher placental miR-146a and miR-182 expression associated with better quality of movement score. We have previously demonstrated that exposure to cigarette smoking in utero leads to downregulation of $m i R$ $146 a$ (8). Taken together with these findings, our results suggest that tobacco smoke exposure may elicit some of its negative effects, such as those reported on newborn muscle tone (22) and overall neurobehavior, through alteration of miRNA expression.

Previous groups have used a variety of in silico target prediction strategies coupled with gold-standard approaches to empirically validate miRNA targets $(5,8,12,17)$, thereby attempting to investigate the mechanisms by which such miRNAs may regulate key cell and developmental processes. Although previous work has suggested that the expression of miR-16 in the placenta is associated with maternal cigarette smoking during pregnancy (8) and fetal growth (12), we are unaware of any reported findings investigating placental $m i R$ 16 expression and infant neurobehavior, more specifically infant attention. Recently, miR-16 was shown to regulate serotonin transporter (SERT) levels. Studies have demonstrated that miR-16 is expressed at relatively higher levels in noradrenergic than in serotonergic cells, with reduction of $m i R-16$ expression in noradrenergic neurons leading to de novo SERT expression (23). Dysregulated signaling through the serotonin pathway associated with prenatal cocaine exposure and prenatal stress has been reported to increase behavioral disturbances in attention, emotional behavior, stress response, and other outcomes (24). We have recently shown that intrauterine exposure to as modest of an alteration as a change in maternal mood is associated with increased placental SERT expression (25). Given that miR-16 targets SERT messenger RNA, increased miR-16 expression may lead to decreased levels of SERT, thereby dysregulating signaling through the serotonin 
Table 4. Predicted mRNA targets of miR-182

\begin{tabular}{lc}
\hline miRNA & Predicted target mRNA \\
\hline miR-182 & PRKACB \\
& PCDH8 \\
& CTTN \\
& EPAS1 \\
\hline
\end{tabular}

Targets for miR-182 were predicted using a modified target prediction strategy (ref. 8). Three target prediction algorithms, miRanda (August 2010 release, http://www. microrna.org/microrna/home.do), PicTar ((as cited in ref. 40), http://pictar.mdc-berlin. de/cgi-bin/new_PicTar_vertebrate.cgi), and TargetScan 6.1 (http://www.targetscan. org/) were used to predict targets for miR-182. To be considered a predicted target, the target must have appeared in the top 100 targets in all three prediction algorithms.

CTTN, cortactin; EPAS1, endothelial PAS domain protein 1; mRNA, messenger RNA; miRNA, microRNA; PCDH8, protocadherin 8; PRKACB, cyclic adenosine monophosphate-dependent protein kinase catalytic subunit $\beta$.

pathway, which may lead to disrupted attention. This may partially explain the negative association we found between $m i R$ 16 expression in the placenta and infant attention score. Future research in both human cohorts and animal models is needed to more definitively test this hypothesis.

Previously reported target prediction analysis (8), coupled with empirical validation (26), has demonstrated TRAF6 as a target of $m i R-146 a$. This suggests that $m i R-146 a$ can influence signaling through the NFk $\beta$ pathway. Previous work has reported that TRAF6 deficiency can result in exencephaly and plays a role in promoting programmed cell death in the developing central nervous system (27). Future work using model systems to more comprehensively investigate how dysregulated placental miR-146a expression may influence the developing central nervous system will be important in furthering our understanding of quality of movement in infants.

Differential expression of $m i R-182$ in the placentas of patients with preeclampsia has been described (7). One of the targets of miR-182 is FOXO1 (28), a transcription factor important for regulating genes in the pathways regulating cell cycle checkpoints, apoptosis, and metabolism. Using a previously described in silico target prediction strategy (8), we further predicted targets for $m i R-182$ to include cyclic adenosine monophosphatedependent protein kinase catalytic subunit $\beta$ (PRKACB), protocadherin 8 , cortactin, and endothelial PAS domain protein 1). Table 4 summarizes these findings. PRKACB is a member of the Ser/Thr protein kinase family and a catalytic subunit of protein kinase A (PKA), which has been previously shown to interact with the ryanodine receptor 2 (29) and the low-affinity nerve growth factor receptor (30). Previous work has suggested that PKA is involved in dopamine signaling in cells and furthermore that PKA inhibition blocks the acquisition but not expression of amphetamine-produced conditioning in amphetamine-dosed rats (31). If PRKACB is a target of $m i R-182$, increased $m i R-182$ expression might lead to decreased levels of PRKACB, which may have consequences for a number of cellular processes, including dysregulated activation of the reward system in neurons in the nucleus accumbens. In the placenta, cross-talk between the PKA and the mitogen-activated protein kinase (MAPK) signaling pathways can mediate placental leptin expression, thereby having an effect on the proliferation and survival of trophoblast cells (32). Protocadherin 8 is the human ortholog of paraxial protocadherin (PAPC), an important mediator during vertebrate embryogenesis $(33,34)$. Cortactin is present in many cell types and, when activated, plays an important role in promoting cell migration and in lamellipodia and invadopodia formation (35-37). Initial reports have investigated endothelial PAS domain protein 1 levels in placentas associated with preeclampsia and hypoxia (38), finding that endothelial PAS domain protein 1 was upregulated in preeclamptic placentas. Future work to empirically validate PRKACB, protocadherin 8 , cortactin, and endothelial PAS domain protein 1 as targets of $m i R-182$ will be necessary to better elucidate the mechanisms altered by dysregulated placental miR-182 expression.

A growing body of literature is also describing how the placenta and placental gene expression is crucial for proper growth and neurodevelopment of the fetus. Many of the genes implicated as predicted and/or empirically validated targets of $m i R-16, m i R-146 a$, and $m i R-182$ are involved in placental invasion into the maternal decidua as well as ensuring proper functioning of the placenta during development. Improper invasion or anchoring of the placenta has been hypothesized to increase risk for hypoxia, which, along with other prenatal stressors, can lead to both intrauterine growth restriction as well as changes to the hippocampus in the infant (39). Our work also suggests that the miRNA may be affecting key neuroactive peptide and hormone production by the placenta. These alterations may be additional mode(s) through which the environment can have an impact on infant neurobehavioral development.

Our work is limited by an inability to definitively test causality. The associations we have observed may be further tested using model systems. Another limitation of this study is the relatively moderate sample size for examining the number of neurobehavioral outcomes as determined by the NNNS assessment. To date, our follow-up of the infants studied is limited. Therefore, more extensive associations between miRNA expression and long-term neurobehavioral outcomes remain to be elucidated (15). In addition, our samples were limited to term placentas, which may not accurately represent the changes in placental miRNA expression throughout the various stages in pregnancy. Due to this limitation, we cannot properly speculate about associations between placental miRNA expression and neurobehavioral outcomes in premature infants. Future directions also include performing our analysis in a larger set of mother-infant pairs to examine more complex interactions between placental miRNA expression and infant neurobehavioral outcomes.

In summary, this study reveals associations between placental miRNA expression and infant neurobehavioral outcomes, as measured by the NNNS. The predictive nature of these early neurobehavioral measures has been validated and thus warrants further follow-up of these infants to more definitively determine the association of placental miRNA expression with longer-term neurobehavioral outcomes. Future studies focused on enhancing our understanding of the influence of the prenatal environment on neurobehavioral outcomes through epigenetic mechanisms will be important in elucidating the developmental origins of health and neurobehavior. 


\section{METHODS}

\section{Study Population}

Study subjects are part of the ongoing Rhode Island Child Health Study, in which mother-infant pairs are enrolled following delivery at Women \& Infants Hospital of Rhode Island $(12,15,16)$. In brief, term infants born small for gestational age (lowest 10th percentile), or large for gestational age (highest 10th percentile) based on birth weight and gestational age and calculated using the Fenton growth chart (19) are selected. Appropriate for gestational age infants matched on gender, gestational age $( \pm 3 \mathrm{~d})$, and maternal age $( \pm 2$ y) are also enrolled. Only viable, singleton infants are included. Additional exclusion criteria include congenital or chromosomal abnormality of the infant, maternal age $<18 \mathrm{y}$ or $>40 \mathrm{y}$, and a life-threatening complication of the mother. Data from maternal inpatient medical records from delivery were collected by structured chart review. Mothers were subjected to an interviewer-administered questionnaire including questions regarding exposure histories, lifestyle, and demographics. All subjects provided written informed consent for participation under appropriate protocols approved by the institutional review boards for Women \& Infants' Hospital of Rhode Island and Brown University.

\section{Neurobehavioral Assessment}

Certified psychometrists, blinded to the study hypothesis, administered the NNNS during the newborn inpatient stay and before discharge. The NNNS assessments, as well as established summary scores, have been described previously $(18,20)$. This analysis examined 86 subjects enrolled between September 2009 and September 2010 having complete NNNS data, placental miRNA expression profiling, and demographics information.

\section{Placenta Sample Collection and RNA Extraction}

The placenta sample collection and RNA extraction have been described previously $(12,16)$. For each patient, 12 samples of placenta tissue, three from each of four quadrants (totaling $\sim 1 \mathrm{~g}$ of tissue) were excised from the placenta within $2 \mathrm{~h}$ of delivery. All samples were taken from the maternal side of the placenta, $2 \mathrm{~cm}$ from the umbilical cord insertion site, free of maternal decidua. Immediately after sample collection, the samples were placed in RNAlater (Ambion/Life Technologies, Carlsbad, CA) and stored at $4{ }^{\circ} \mathrm{C}$. At least $72 \mathrm{~h}$ later, placenta samples were removed from RNAlater, blotted dry, snapfrozen in liquid nitrogen, and homogenized using a mortar and pestle to create a single homogenized sample, thereby reducing variability introduced by sampling site. The samples were stored at $-80^{\circ} \mathrm{C}$ until needed for further examination. RNA was extracted from the homogenized samples using the miRvana miRNA Isolation Kit (Ambion/ Life Technologies) and manufacturer protocols as described previously $(8,12)$.

\section{Quantitative Real-Time PCR for Mature miRNA}

Absolute quantitation of the expression of mature miRNA was measured using TaqMan miRNA Assays (Applied Biosystems, Valencia, CA) on an Applied Biosystems 7900HT Real-Time PCR system and analyzed with 7900HT System Software (Applied Biosystems) as described previously (12). All reactions were run in triplicate on 384well plates, and RNU-44 was used as an internal control to check that starting concentrations were standardized. For the 86 samples, the mean threshold cycle (Ct) value of RNU-44 was 24.72, and the SD was 0.62 , suggesting highly standardized starting concentrations, and allowing use of the absolute quantification in subsequent analyses. No-reverse-transcription controls were run to assure samples were free of genomic DNA contamination..

\section{Statistical Analysis}

Because miRNA expression profiles were not normally distributed as reported previously (12), bivariate associations between NNNS summary scores and quartiles of miRNA expression were assessed using nonparametric Kruskal-Wallis tests. Kruskal-Wallis tests were used to assess differences in all six NNNS summary scores across quartiles of miRNA expression, and $P<0.05$ was used to indicate a significant difference in respective NNNS summary scores across quartiles of respective miRNA expression. We focused on six summary scores shown to be the most sensitive on the basis of our preliminary studies, specifically attention, quality of movement, arousal, excitability, hypertonicity, and stress abstinence (20). Linear regression models were used to investigate associations between NNNS summary scores and miRNA expression while controlling for confounders that may influence this association. Final models of infant attention scores and quality of movement scores and miRNA expression included infant gender, maternal age, maternal insurance, relative maternal weight gained during pregnancy, maternal smoking during pregnancy, delivery method, and birth weight percentile, thereby controlling for potential confounders. All statistical analyses were performed in R ( R Version 2.12.0; The R Foundation for Statistical Computing, Vienna, Austria).

\section{In Silico miRNA Target Prediction}

Targets for miRNAs of interest were predicted using a modified target prediction strategy as described previously (8). Three target prediction algorithms accessible online, miRanda (August 2010 release), PicTar (as cited in ref. 40), and TargetScan 6.1, were used to predict targets for miRNAs of interest. To be considered a predicted target, the target must have appeared in the top 100 targets in all three prediction algorithms.

\section{SUPPLEMENTARY MATERIAL}

Supplementary material is linked to the online version of the paper at http:// www.nature.com/pr

\section{ACKNOWLEDGMENTS}

The authors thank Gilda Ferro and Joyce Lee for their work in recruitment of subjects into the study and the staff of the Brown Center for the Study of Children at Risk for their efforts.

\section{STATEMENT OF FINANCIAL SUPPORT}

This work was supported by National Institutes of Health (Bethesda, MD) grants from the National Center for Research Resources (P20 RR018728), the National Institute of Mental Health (R01 MH094609), and the National Institute of Alcohol Abuse and Alcoholism (2 T32 AA 07459-26, to M.A.M.). The funders had no role in the study design, data collection and analysis, decision to publish, or preparation of the manuscript.

Disclosure: The authors have no conflict of interest to declare.

\section{REFERENCES}

1. Barker DJ. Fetal programming of coronary heart disease. Trends Endocrinol Metab 2002;13:364-8.

2. Van den Bergh BR. Developmental programming of early brain and behaviour development and mental health: a conceptual framework. Dev Med Child Neurol 2011;53:Suppl 4:19-23.

3. Yen SS. The placenta as the third brain. J Reprod Med 1994;39:277-80.

4. Lester BM, Padbury JF. Third pathophysiology of prenatal cocaine exposure. Dev Neurosci 2009;31:23-35.

5. Maccani MA, Marsit CJ. Epigenetics in the placenta. Am J Reprod Immunol 2009;62:78-89.

6. Miska EA. How microRNAs control cell division, differentiation and death. Curr Opin Genet Dev 2005;15:563-8.

7. Pineles BL, Romero R, Montenegro D, et al. Distinct subsets of microRNAs are expressed differentially in the human placentas of patients with preeclampsia. Am J Obstet Gynecol 2007;196:261.e1-6.

8. Maccani MA, Avissar-Whiting M, Banister CE, McGonnigal B, Padbury JF, Marsit CJ. Maternal cigarette smoking during pregnancy is associated with downregulation of miR-16, miR-21, and miR-146a in the placenta. Epigenetics 2010;5:583-9.

9. Avissar-Whiting M, Veiga KR, Uhl KM, et al. Bisphenol A exposure leads to specific microRNA alterations in placental cells. Reprod Toxicol 2010;29:401-6.

10. Chim SS, Shing TK, Hung EC, et al. Detection and characterization of placental microRNAs in maternal plasma. Clin Chem 2008;54:482-90.

11. Mouillet JF, Chu T, Hubel CA, Nelson DM, Parks WT, Sadovsky Y. The levels of hypoxia-regulated microRNAs in plasma of pregnant women with fetal growth restriction. Placenta 2010;31:781-4. 
12. Maccani MA, Padbury JF, Marsit CJ. miR-16 and miR-21 expression in the placenta is associated with fetal growth. PLoS ONE 2011;6:e21210.

13. Kocerha J, Faghihi MA, Lopez-Toledano MA, et al. MicroRNA-219 modulates NMDA receptor-mediated neurobehavioral dysfunction. Proc Natl Acad Sci USA 2009;106:3507-12.

14. Li MD, van der Vaart AD. MicroRNAs in addiction: adaptation's middlemen? Mol Psychiatry 2011;16:1159-68.

15. Marsit CJ, Lambertini L, Maccani MA, et al. Placenta-imprinted gene expression association of infant neurobehavior. J Pediatr 2012;160:854860.e2.

16. Marsit CJ, Maccani MA, Padbury JF, Lester BM. Placental 11-beta hydroxysteroid dehydrogenase methylation is associated with newborn growth and a measure of neurobehavioral outcome. PLoS ONE 2012;7:e33794.

17. Maccani MA, Marsit CJ. Exposure and fetal growth-associated miRNA alterations in the human placenta. Clin Epigenetics 2011;2:401-4.

18. Lester BM, Tronick EZ, Brazelton TB. The Neonatal Intensive Care Unit Network Neurobehavioral Scale procedures. Pediatrics 2004;113(3 Pt 2):641-67.

19. Fenton TR. A new growth chart for preterm babies: Babson and Benda's chart updated with recent data and a new format. BMC Pediatr 2003;3:13.

20. Liu J, Bann C, Lester B, et al. Neonatal neurobehavior predicts medical and behavioral outcome. Pediatrics 2010;125:e90-8.

21. Boukydis CF, Bigsby R, Lester BM. Clinical use of the Neonatal Intensive Care Unit Network Neurobehavioral Scale. Pediatrics 2004;113(3 Pt 2):679-89.

22. Stroud LR, Paster RL, Goodwin MS, et al. Maternal smoking during pregnancy and neonatal behavior: a large-scale community study. Pediatrics 2009;123:e842-8.

23. Baudry A, Mouillet-Richard S, Schneider B, Launay JM, Kellermann O. miR-16 targets the serotonin transporter: a new facet for adaptive responses to antidepressants. Science 2010;329:1537-41.

24. Williams SK, Lauder JM, Johns JM. Prenatal Cocaine Disrupts Serotonin Signaling-Dependent Behaviors: Implications for Sex Differences, Early Stress and Prenatal SSRI Exposure. Curr Neuropharmacol 2011;9:478-511.

25. Ponder KL, Salisbury A, McGonnigal B, Laliberte A, Lester B, Padbury JF. Maternal depression and anxiety are associated with altered gene expression in the human placenta without modification by antidepressant use: implications for fetal programming. Dev Psychobiol 2011;53:711-23.

26. Hou J, Wang P, Lin L, et al. MicroRNA-146a feedback inhibits RIG-Idependent Type I IFN production in macrophages by targeting TRAF6, IRAK1, and IRAK2. J Immunol 2009;183:2150-8.
27. Lomaga MA, Henderson JT, Elia AJ, et al. Tumor necrosis factor receptor-associated factor 6 (TRAF6) deficiency results in exencephaly and is required for apoptosis within the developing CNS. J Neurosci 2000;20:7384-93.

28. Guttilla IK, White BA. Coordinate regulation of FOXO1 by miR-27a, miR96, and miR-182 in breast cancer cells. J Biol Chem 2009;284:23204-16.

29. Marx SO, Reiken S, Hisamatsu Y, et al. PKA phosphorylation dissociates FKBP12.6 from the calcium release channel (ryanodine receptor): defective regulation in failing hearts. Cell 2000;101:365-76.

30. Higuchi H, Yamashita T, Yoshikawa H, Tohyama M. PKA phosphorylates the p75 receptor and regulates its localization to lipid rafts. EMBO J 2003;22:1790-800.

31. Gerdjikov TV, Giles AC, Swain SN, Beninger RJ. Nucleus accumbens PKA inhibition blocks acquisition but enhances expression of amphetamine-produced conditioned activity in rats. Psychopharmacology (Berl) 2007;190:65-72.

32. Maymó JL, Pérez AP, Gambino Y, Calvo JC, Sánchez-Margalet V, Varone CL. Review: Leptin gene expression in the placenta-regulation of a key hormone in trophoblast proliferation and survival. Placenta 2011;32:Suppl 2:S146-53.

33. Kim SH, Yamamoto A, Bouwmeester T, Agius E, Robertis EM. The role of paraxial protocadherin in selective adhesion and cell movements of the mesoderm during Xenopus gastrulation. Development 1998;125:4681-90.

34. Rhee J, Takahashi Y, Saga Y, Wilson-Rawls J, Rawls A. The protocadherin papc is involved in the organization of the epithelium along the segmental border during mouse somitogenesis. Dev Biol 2003;254:248-61.

35. Weed SA, Parsons JT. Cortactin: coupling membrane dynamics to cortical actin assembly. Oncogene 2001;20:6418-34.

36. Weaver AM, Karginov AV, Kinley AW, et al. Cortactin promotes and stabilizes Arp2/3-induced actin filament network formation. Curr Biol 2001;11:370-4.

37. Ammer AG, Weed SA. Cortactin branches out: roles in regulating protrusive actin dynamics. Cell Motil Cytoskeleton 2008;65:687-707.

38. Jarvenpaa J, Vuoristo JT, Savolainen ER, Ukkola O, Vaskivuo T, Ryynanen M. Altered expression of angiogenesis-related placental genes in preeclampsia associated with intrauterine growth restriction. Gynecol Endocrinol 2007;23:351-5.

39. Lodygensky GA, Seghier ML, Warfield SK, et al. Intrauterine growth restriction affects the preterm infant's hippocampus. Pediatr Res 2008;63:438-43.

40. Lall S, Grün D, Krek A, et al. A genome-wide map of conserved microRNA targets in C. elegans. Curr Biol 2006;16:460-71. 